\begin{tabular}{|c|l|}
\hline Title & Gauge theory on a non-simply connected domain and representations of canonical commutation relations \\
\hline Author(s) & A rai, A sao \\
\hline Citation & Journal of Mathematical Physics, 36(6), 2569-2580 \\
\hline https://doi.org/10.1063/1.531051 \\
\hline Issue Date & 1995-06 \\
\hline Doc URL & http://hdl.handle.net/2115/13684 \\
\hline Rights & Copyright $\odot 1995$ A merican Institute of Physics \\
\hline Type & article \\
\hline File Information & jmp36-6.pdf \\
\hline
\end{tabular}

Instructions for use 


\title{
Gauge theory on a non-simply connected domain and representations of canonical commutation relations
}

\author{
Asao Arai \\ Department of Mathematics, Hokkaido University, Sapporo 060, Japan
}

(Received 17 January 1995; accepted for publication 10 February 1995)

\begin{abstract}
A quantum system of a particle interacting with a (non-Abelian) gauge field on the non-simply connected domain $M=\mathbf{R}^{2}\left\{\mathbf{a}_{n}\right\}_{n=1}^{N}$ is considered, where $\mathbf{a}_{n}, n=1, \ldots, N$, are fixed isolated points in $\mathbf{R}^{2}$. The gauge potential $A$ of the gauge field is a $p \times p$ anti-Hermitian matrix-valued 1 -form on $M$, and may be strongly singular at the points $\mathbf{a}_{i 2}, n=1, \ldots, N$. If $A$ is flat, then the physical momentum and the position operators $\left\{P_{j}, q_{j}\right\}_{j=1}^{2}$ of the particle satisfy the canonical commutation relations (CCR) with two degrees of freedom on a suitable dense domain of the Hilbert space $L^{2}\left(\mathbf{R}^{2} ; \mathbf{C}^{p}\right)$. A necessary and sufficient condition for this representation to be the Schrödinger 2-system is given in terms of the Wilson loops of the rectangles not intersecting $\mathrm{a}_{n}, n=1, \ldots, N$. This also gives a characterization for the representation to be non-Schrödinger. It is proven that, for a class of gauge potentials, which is not necessarily flat, $P_{j}$ is essentially self-adjoint. Morcover, an cxample, which gives a class of non-Schrödinger representations of the CCR with two degrees of freedom, is discussed in some detail. (C) 1995 American Institute of Physics.
\end{abstract}

\section{INTRODUCTION}

In Ref. 1 , the author considered a quantum system of a charged particle with charge $q \in \mathbf{R} \backslash\{0\}$ moving in the plane $\mathbf{R}^{2}$ under the influence of a perpendicular magnetic field, which may be singular at given fixed isolated points $\mathbf{a}_{n}=\left(a_{n 1}, a_{n 2}\right) \in \mathbf{R}^{2}, n=1, \ldots, N$. In this quantum system, the position operators $q_{j}$ and the physical momentum operators $P_{j}=p_{j}-q A_{j}(j=1,2)$, where $p_{j}$ is the canonical momentum operator with respect to $q_{j}$ and $\left(A_{1}, A_{2}\right)$ is a vector potential of the magnetic field, satisfy, on a dense domain of $L^{2}\left(\mathbf{R}^{2}\right)$, the canonical commutation relations (CCR) with two degrees of freedom if and only if the magnetic field is concentrated on the set $\left\{\mathbf{a}_{n}\right\}_{n=1}^{N}$.

A set $\left\{\hat{P}_{1}, \ldots, \hat{P}_{n} ; \hat{Q}_{1}, \ldots, \hat{Q}_{n}\right\}$ of self-adjoint operators on a Hilbert space $\mathscr{W}$ is called a Schrödinger $n$-system if there exist mutually orthogonal closed subspaces $\mathscr{H}_{\alpha}$ of $\mathscr{H}_{\text {, such that }}$ $\mathscr{W}=\oplus_{\alpha} \mathscr{H}_{\alpha}$ with the following properties: (i) each $\mathscr{H}_{\alpha}$ reduces all $\hat{P}_{j}, \hat{Q}_{j} ;$ (ii) the set $\left\{\hat{P}_{1}, \ldots, \hat{P}_{n} ; \hat{Q}_{1}, \ldots, \hat{Q}_{n}\right\}$ is, in each $\mathscr{H}_{\alpha}$, irreducible and unitarily equivalent to the Schrödinger system $\left\{P_{1}^{s}, \ldots, P_{n}^{s} ; Q_{1}^{s}, \ldots, Q_{n}^{s}\right\}$, where $P_{j}^{s}$ and $Q_{j}^{s}$ are self-adjoint operators on $L^{2}\left(\mathbf{R}^{n}\right)$ defined by $P_{j}^{s}=-i \partial_{j}\left[\partial_{j}\right.$ denotes the generalized partial differential operator in the $j$ th variable $x_{j}$ of $\left.\mathrm{x}=\left(x_{1}, \ldots, x_{n}\right) \in \mathbf{R}^{n}\right]$ and $Q_{j}^{s}=x_{j}$ (the multiplication operator by $\left.x_{j}\right)$ (Ref. 2, p. 81). We call a representation of the CCR with $n$ degrees of freedom non-Schrödinger if it is not a Schrödinger $n$-system.

It was proven in Ref. 1 that the above mentioned representation $\left\{\bar{P}_{j}, q_{j}\right\}_{j=1}^{2}$ of CCR, where $\bar{P}_{j}$ denotes the closure of $P_{j}$, is a Schrödinger 2-system if and only if the magnetic flux is locally quantized [i.e., the magnetic flux of every rectangle not intersecting $\mathbf{a}_{n}(n=1, \ldots, N)$ is an integer multiple of $2 \pi / q$ ]. This result, which generalizes the main result of Ref. 3 concerning a special example in the case $N=1$, shows that, if the magnetic field is concentrated on $\left\{\mathbf{a}_{n}\right\}_{n=1}^{N}$ and the magnetic flux is not locally quantized, then $\left\{\bar{P}_{j}, q_{j}\right\}_{j=1}^{2}$ is a non-Schrödinger representation of $\mathrm{CCR}$. Thus, a class of non-Schrödinger representations of CCR is constructed. Of interest concerning these non-Schrödinger representations is their relation to the Aharonov-Bohm effect ${ }^{4}$ in an idealized sense. From a mathematical point of view, the non-simply connectedness of the base manifold, 


$$
M=\mathbf{R}^{2} \backslash\left\{\mathbf{a}_{n}\right\}_{n=1}^{N},
$$

is essential for the analysis just mentioned to be non-trivial. Further properties of the quantum system were investigated in terms of the Dirac-Weyl operator. ${ }^{5}$

An operator-theoretical analysis related to Refs. 1 and 3 has been made by Kurose and Nakazato, ${ }^{6}$ who have constructed a $*$ representation of the Weyl algebra with two degrees of freedom induced by a one-dimensional representation of the fundamental group of $M$, and proved that the * representation is unitarily equivalent to the * algebra generated by $\left\{P_{j}, q_{j}\right\}_{j=1}^{2}$.

The quantum system discussed in Ref. 1 is an example of Abelian gauge theory with gauge group $U(1)$, the one-dimensional unitary group. It is natural and interesting to extend the analysis made in Ref. 1 to the case of non-Abelian gauge theory. With this motivation, we consider in this paper a quantum mechanical particle moving in $M$ under the influence of a (non-Abelian) gauge field. Indeed, in this case too, the position and the physical momentum operators of the particle give a representation of the CCR with two degrees of freedom if the gauge field strength is concentrated on $\left\{\mathbf{a}_{n}\right\}_{n=1}^{N}$. We formulate a necessary and sufficient condition for the representation to be a Schrödinger 2-system. As in the case of Ref. 1, this result gives a class of non-Schrödinger representations of CCR, which may give a form of non-commutative Aharonov-Bohm effect. Our analysis is general, in that it applies to any gauge theory on $M$ with a finite-dimensional unitary representation of a Lie group. The Dirac-Weyl operator in the present case will be discussed in a separate paper.

In Sec. II, under the assumption that the physical momentum operators $P_{1}, P_{2}$ are essentially self-adjoint, we compute the commutation relations (in the strong sense) of the position operators $q_{1}, q_{2}$ and $\bar{P}_{1}, \bar{P}_{2}$. It is shown that $\left\{\bar{P}_{j}, q_{j}\right\}_{j=1}^{2}$ is a Schrödinger 2 -system if and only if the Wilson loop of every rectangle not intersecting $\mathbf{a}_{n}(n=1, \ldots, N)$ is equal to the identity. In Sec. III, we derive, in terms of the gauge potential, a condition equivalent to the condition for the Wilson loops just mentioned. This can be done by employing the theory of product integrals. Section IV is devoted to the proof of essential self-adjointness of $P_{j}$ for a wide class of gauge potentials. In the last section we discuss an example in some detail.

\section{CCR IN (NON-ABELIAN) GAUGE THEORY}

Let $M$ be given by (1.1). We denote by $M_{p}^{\text {ah }}(\mathbf{C})$ the set of $p \times p$ anti-Hermitian matrices $(p \in \mathbf{N})$. Let $A_{j}, j=1,2$, be $M_{p}^{\text {ah }}(\mathbf{C})$-valued continuously differcntiablc functions on $M$, and set

$$
A(\mathbf{r})=A_{1}(\mathbf{r}) d x+A_{2}(\mathbf{r}) d y, \quad \mathbf{r}=(x, y) \in M,
$$

an $M_{p}^{\text {ah }}(\mathbf{C})$-valued 1-form on $M$. This 1-form may be regarded as a gauge potential in a gauge theory on $M$ with a $p$-dimensional unitary representation of a Lie group.

We shall use the system of physical units where $\hbar$, the Planck constant divided by $2 \pi$, is equal to 1 . Let $\partial_{1}$ and $\partial_{2}$ be the generalized partial differential operators in $x$ and $y$, respectively, and set

$$
p_{j}=-i \partial_{j}, \quad j=1,2 .
$$

Then the physical momentum operator of a quantum mechanical particle interacting with the gauge potential $A$ may be given by $\mathbf{P}=\left(P_{1}, P_{2}\right)$, with

$$
P_{j}=p_{j}-i A_{j}, \quad j=1,2,
$$

acting in the Hilbert space $L^{2}\left(\mathbf{R}^{2} ; \mathbf{C}^{p}\right)\left[\simeq L^{2}\left(M ; \mathbf{C}^{p}\right)\right]$ of $\mathbf{C}^{p}$-valued square integrable functions on $\mathbf{R}^{2}$. We denote by $C_{0}^{m}\left(M ; \mathbf{C}^{p}\right)$ the set of $\mathbf{C}^{p}$-valued $m$ times continuously differentiable functions on $M$ with compact support. Each $P_{j}$ is a symmetric operator with $C_{0}^{2}\left(M ; C^{p}\right) \subset D\left(P_{1} P_{2}\right) \cap D\left(P_{2} P_{1}\right)$, and 


$$
\left[P_{1}, P_{2}\right] \psi=-F_{12} \psi, \quad \psi \in C_{0}^{2}\left(M ; \mathbf{C}^{p}\right)
$$

where

$$
F_{12}:=\partial_{1} A_{2}-\partial_{2} A_{1}+\left[A_{1}, A_{2}\right]
$$

is the component of the gauge field strength 2-form,

$$
F(A):=d A+A \wedge A=F_{12} d x \wedge d y .
$$

We say that $A$ is flat on $M$ if $F(A)=0$ on $M$.

We denote by $q_{1}$ and $q_{2}$ the multiplication operators by $x$ and $y$, respectively. The following proposition is easily proven.

Proposition 2.1: Suppose that $A$ is flat on $M$. Then $\left\{P_{j}, q_{j}\right\}_{j=1}^{2}$ satisfies the CCR with two degrees of freedom on $C_{0}^{2}\left(M ; \mathbf{C}^{p}\right)$ : for all $\psi \in C_{0}^{2}\left(M ; \mathbf{C}^{p}\right)$,

$$
\left[P_{j}, P_{k}\right] \psi=0, \quad\left[q_{j}, q_{k}\right] \psi=0, \quad\left[q_{j}, P_{k}\right] \psi=i \delta_{j k} \psi, \quad j, k=1,2
$$

For a continuous, piecewise continuously differentiable path $C$ in $M$ with parametrization $\gamma(\tau)=\left(\gamma_{1}(\tau), \gamma_{2}(\tau)\right), \tau \in[a, b](a<b, a, b \in \mathbf{R})$, we define the Wilson loop $W_{A}(C)$ by

$$
W_{A}(C)=\prod_{a}^{b} e^{-\left\{A_{1}(\gamma(\tau)) \dot{\gamma}_{1}(\tau)+A_{2}(\gamma(\tau)) \dot{\gamma}_{2}(\tau)\right\} d \tau}
$$

where $\dot{\gamma}_{j}(\tau)=d \gamma_{j}(\tau) / d \tau, j=1,2$, and the right-hand side (RHS) is the product integral of the matrix-valued function, $-\left\{A_{1}(\gamma(\tau)) \dot{\gamma}_{1}(\tau)+A_{2}(\gamma(\tau)) \dot{\gamma}_{2}(\tau)\right\}$ on the interval $[a, b]$ (see Rer. 7, Sec. 1.1). ${ }^{8}$ It can be shown that $W_{A}(C)$ is independent of the choice of parametrizations of $C$ (cf. Ref. 7. Sec. 2.1). It follows from the anti-Hermiticity of $A_{j}$ that $W_{A}(C)$ is in $U(p)$, the set of $p \times p$ unitary matrices.

For $x, y^{y}, s, t \in \mathbf{R}$, we define two hook-shaped paths $C_{x, y, s, t}^{ \pm}$from $(x, y)$ to $(x+s, y+t)$, with parametrizations $\gamma_{x, y, s, t}^{ \pm}:[0,1] \rightarrow \mathbf{R}^{2}$ given by

$$
\begin{aligned}
& \gamma_{x, y ; s, t}^{-}(\tau)=\left\{\begin{array}{l}
(x+2 \tau s, y), \quad 0 \leqslant \tau \leqslant \frac{1}{2}, \\
(x+s, y+(2 \tau-1) t), \quad \frac{1}{2} \leqslant \tau \leqslant 1 ;
\end{array}\right. \\
& \gamma_{x, y ; s, t}^{+}(\tau)= \begin{cases}(x, y+2 \tau t), & 0 \leqslant \tau \leqslant \frac{1}{2}, \\
(x+(2 \tau-1) s, y+t), & \frac{1}{2} \leqslant \tau \leqslant 1 ;\end{cases}
\end{aligned}
$$

and set

$$
C_{x, y ; s, t}=\left\{C_{x, y ; s, t}^{+}\right\}^{-1} \circ C_{x, y ; s, t}^{-},
$$

which is the rectangle: $(x, y) \rightarrow(x+s, y) \rightarrow(x+s, y+t) \rightarrow(x, y+t) \rightarrow(x, y)$.

For each $s, t \in \mathbf{R}$, let

$$
\mathbf{R}_{s}^{(1)}=\mathbf{R} \backslash\left\{a_{n 1}, a_{n 1}-s\right\}_{n=1}^{N}, \quad \mathbf{R}_{t}^{(2)}=\mathbf{R} \backslash\left\{a_{n 2}, a_{n 2}-t\right\}_{n=1}^{N},
$$

and $M_{s, t}=\mathbf{R}_{s}^{(1)} \times \mathbf{R}_{t}^{(2)}$. If $(x, y) \in M_{s, t}$, then $C_{x, y, s, t}^{ \pm}$do not intersect $\mathbf{a}_{n}, n=1, \ldots, N$. Hence, for each $s, t \in \mathbf{R}$, we can define $U(p)$-valued functions $W_{s, t}^{A, \pm}, W_{s, t}^{A}$ on $M_{s, t}$ by

$$
W_{s, t}^{A, \pm}(x, y)=W_{A}\left(C_{x, y ; s, t}^{ \pm}\right), \quad W_{s, t}^{A}(x, y)=W_{A}\left(C_{x, y ; s, t}\right), \quad(x, y) \in M_{s, t} .
$$


The two-dimensional Lebesgue measure of the set $\mathbf{R}^{2} \backslash M_{s, t}$ is zero. Hence, $W_{s, t}^{A, \pm}$ and $W_{s, t}^{A}$ can be regarded as almost everywhere (a.e.) finite $U(p)$-valued functions on $\mathbf{R}^{2}$, so that they define unitary operators on $L^{2}\left(\mathbf{R}^{2} ; \mathbf{C}^{p}\right)$ as multiplication operators. We denote these unitary multiplication operators by the same symbols $W_{s, t}^{A, \pm}, W_{s, t}^{A}$, respectively.

Throughout the rest of this section, we assume the following.

Assumption: The operators $P_{1}$ and $P_{2}$ are essentially self-adjoint.

Theorem 2.2: For all $s, t \in \mathbf{R}$,

$$
e^{i s \bar{P}_{1}} e^{i t \bar{P}_{2}}=\left(W_{s, t}^{A}\right) * e^{i t \bar{P}_{2}} e^{i s \bar{P}_{1}}
$$

Proof: The method of proof is similar to the proof of Theorem 2.1 in Ref. 1. By the present assumption, we can apply the Trotter product formula to obtain

$$
\left\langle\phi, e^{i t \bar{P}_{j}} \psi\right\rangle=\lim _{m \rightarrow \infty}\left\langle\phi,\left(e^{i t p_{j} / m} e^{t A_{j} / m}\right)^{m} \psi\right\rangle,
$$

where $\langle\cdot, \cdot\rangle$ denotes the inner product of $L^{2}\left(\mathbf{R}^{2} ; \mathbf{C}^{p}\right)$. We denote by $L_{(x, y) ;\left(x^{\prime}, y^{\prime}\right)}$ the straight line from $(x, y)$ to $\left(x^{\prime}, y^{\prime}\right)$. The straight line $L_{(x+s, y) ;(x, y)}$ is parametrized by $\gamma(\tau)=(x+(1-\tau) s, y)$, $\tau \in[0,1]$. Then $\dot{\gamma}(\tau)=-s(1,0)$. In terms of $\gamma(\tau)$ and $\dot{\gamma}(\tau)$, we can write

$$
\begin{aligned}
& \left(\left(e^{i s p_{1} / m} e^{s A_{1} / m}\right)^{m} \psi\right)(x, y) \\
& =\exp \left(-A_{1}\left(\gamma\left(\frac{m-1}{m}\right)\right) \dot{\gamma}_{1}\left(\frac{m-1}{m}\right) \frac{1}{m}\right) \cdots \exp \left(-A_{1}\left(\gamma\left(\frac{1}{m}\right)\right) \dot{\gamma}_{1}\left(\frac{1}{m}\right) \frac{1}{m}\right) \\
& \quad \times \exp \left(-A_{1}(\gamma(0)) \dot{\gamma}_{1}(0) \frac{1}{m}\right) \psi(x+s, y),
\end{aligned}
$$

which converges to $W_{A}\left(L_{(x+s, y) ;(x, y)}\right) \psi(x+s, y)$ a.e. $(x, y) \in M_{s, t}$ as $m \rightarrow \infty$. Moreover, by the fact that $e^{-A_{1}(\gamma(\tau)) \dot{\gamma}_{1}(\tau) / m} \in U(p)$, we have

$$
\left\|\phi(x, y)\left(e^{i s p_{1} / m} e^{s A_{1} / m}\right)^{m} \psi(x, y)\right\|_{\mathbf{C}^{p}} \leqslant\|\phi(x, y)\|_{\mathbf{C}^{p}}\|\psi(x+s, y)\|_{\mathbf{C}^{p}}
$$

and

$$
\int_{\mathbf{R}^{2}}\|\phi(x, y)\|_{\mathbf{C}^{p}}\|\psi(x+s, y)\|_{\mathbf{C}^{p}} d \mathbf{r} \leqslant\left(\int_{\mathbf{R}^{2}}\|\phi(\mathbf{r})\|_{\mathbf{C}^{p}}^{2} d \mathbf{r}\right)^{1 / 2}\left(\int_{\mathbf{R}^{2}}\|\psi(\mathbf{r})\|_{\mathbf{C}^{p}}^{2} d \mathbf{r}\right)^{1 / 2}<\infty
$$

Hence, by the dominated convergence theorem, we obtain for all $\phi, \psi \in L^{2}\left(\mathbf{R}^{2} ; \mathbf{C}^{p}\right)$,

$$
\left\langle\phi, e^{i s \bar{P}_{1}} \psi\right\rangle=\int_{\mathbf{R}^{2}}\left\langle\phi(x, y), W_{A}\left(L_{(x+s, y) ;(x, y)}\right) \psi(x+s, y)\right\rangle_{\mathbf{C}} d \mathbf{r}
$$

which implies that

$$
\left(e^{i s \bar{P}_{1}} \psi\right)(x, y)=W_{A}\left(L_{(x+s, y) ;(x, y)}\right) \psi(x+s, y), \quad \text { a.e. }(x, y) \in \mathbf{R}^{2} .
$$

Similarly, we can show that

$$
\left(e^{i t \bar{P}_{2}} \psi\right)(x, y)=W_{A}\left(L_{(x, y+t) ;(x, y)}\right) \psi(x, y+t), \quad \text { a.e. }(x, y) \in \mathbf{R}^{2} .
$$

Combining these formulas, we obtain 


$$
e^{i s \bar{P}_{1}} e^{i t \bar{P}_{2}}=\left(W_{s, t}^{A,-}\right)^{-1} e^{i s p_{1}} e^{i t p_{2}}, \quad e^{i t \bar{P}_{2}} e^{i s \bar{P}_{1}}=\left(W_{s, t}^{A,+}\right)^{-1} e^{i s p_{1}} e^{i t p_{2}}
$$

which, together with the fact $\left(W_{s, t}^{A,-}\right)^{-1} W_{s, t}^{A,+}=\left(W_{s, t}^{A}\right)^{-1}=\left(W_{s, t}^{A}\right)^{*}$, imply (2.3).

As for the commutation relations of $\exp \left(i s q_{j}\right)$ and $\exp \left(i t \bar{P}_{j}\right)(s, t \in \mathbf{R})$, we have the following.

Lemma 2.3: For all $s, t \in \mathbf{R}$,

$$
e^{i s q_{j}} e^{i t \bar{P}_{k}}=e^{-i s t \delta_{j k}} e^{i t \bar{P}_{k}} e^{i s q_{j}}, \quad j, k=1,2
$$

Proof: Similar to the proof of Theorem 2.1. Note that $\left\{e^{i t q_{j}}, e^{i t p_{j}} \mid t \in \mathbf{R}, j=1,2\right\}$ satisfies the Weyl relations with two degrees of freedom (e.g., Ref. 2, p. 81).

Theorem 2.2 and Lemma 2.3 imply the following.

Theorem 2.4: The set $\left\{e^{i t q_{j}}, e^{i t P_{j}} \mid t \in \mathbf{R}, j=1,2\right\}$ satisfies the Weyl relations with two degrees of freedom if and only if $W_{s, f}^{A}=I$ for all $s, t \in \mathbf{R}$, where $I$ is the identity operator on $L^{2}\left(\mathbf{R}^{2} ; \mathbf{C}^{p}\right)$.

As a corollary, we obtain the following.

Corollary 2.5: Suppose that $A$ is flat on $M$. Then $\left\{\bar{P}_{j}, q_{j}\right\}_{j=1}^{2}$ is a Schrödinger 2-system if and only if $W_{s, t}^{A}=I$ for all $s, t \in \mathbf{R}$.

Proof: We need only apply the well-known fact that a representation of the CCR with $n$ degrees of freedom, satisfying the Weyl relations with the same degrees of freedom, is a Schrödinger $n$-system (von Neumann's theorem, ${ }^{9}$ Ref. 2 , p. 81, Theorem 4.11.1).

\section{THE WILSON LOOPS $W_{s, t}^{A}$}

In view of Corollary 2.5 we derive a condition equivalent to the condition that $W_{s, t}^{A}=I$. Let

$$
\delta_{0}=\min _{n \neq m ; n, m=1, \ldots, N}\left|\mathbf{a}_{n}-\mathbf{a}_{m}\right|
$$

For a positive constant $\epsilon<\delta_{0}$ and $\mathbf{r} \in M$ with $\left|\mathbf{r}-\mathbf{a}_{n}\right|=\epsilon$, we denote by $C_{\epsilon}^{\mathbf{r}}\left(\mathbf{a}_{n}\right)$ the circle of radius $\epsilon$ with center $\mathbf{a}_{n}$ and initial point $\mathbf{r}$, where the direction of the circle is taken to be anticlockwise. In this section we prove the following theorem.

Theorem 3.1: The equality $W_{s, t}^{A}=I$ holds for all $s, t \in \mathbf{R}$ if and only if $A$ is flat on $M$, and there exists a constant $\delta \in\left(0, \delta_{0}\right)$, such that for all $\epsilon<\delta$ and some $\mathbf{r}_{n} \in M$ with $\left|\mathbf{r}_{n}-\mathbf{a}_{n}\right|=\epsilon$,

$$
W_{A}\left(C_{\epsilon}^{\mathbf{r}_{n}}\left(\mathbf{a}_{n}\right)\right)=I, \quad n=1, \ldots, N .
$$

Remark: One can easily show that, if $W_{A}\left(C_{\epsilon}^{\mathbf{r}_{n}}\left(\mathbf{a}_{n}\right)\right)=I$ for some $\mathbf{r}_{n}$ with $\left|\mathbf{r}_{n}-\mathbf{a}_{n}\right|=\epsilon$, then $W_{A}\left(C_{\epsilon}^{\mathbf{r}}\left(\mathbf{a}_{n}\right)\right)==I$ for all $\mathbf{r}$, with $\left|\mathbf{r}-\mathbf{a}_{n}\right|=\epsilon$.

We denote by $D_{x, y ; s, t}$ the interior domain of the closed path $C_{x, y ; s, t}$.

Lemma 3.2: For all $(x, y) \in M$,

$$
W_{t, t}^{A}(x, y)=I-\int_{D_{x, y: t, t}} F_{12}\left(\mathbf{r}^{\prime}\right) d \mathbf{r}^{\prime}+O\left(|t|^{3}\right)
$$

as $t \rightarrow 0$.

Proof: Informal proofs of this lemma can be found in the physics literature (e.g., Ref. 10, pp. 52-53). For the sake of completeness, we give a rigorous proof of it. Let $\gamma_{x, y ; s, t}:[0,1] \rightarrow M$ be the parametrization of the path $C_{x, y ; s, t}$, such that $\gamma_{x, y ; s, t}(\tau)=\gamma_{x, y, s, t}^{-}(2 \tau)$ for $0 \leqslant \tau \leqslant \frac{1}{2}$ and $\gamma_{x, y: s, t}(\tau)=\gamma_{x, y, s, t}^{+}\left(1-2\left(\tau-\frac{1}{2}\right)\right)$ for $1 / 2 \leqslant \tau \leqslant 1$, where $\gamma_{x, y, s, t}^{ \pm}(\tau)$ are given by (2.1) and (2.2). Let

$$
B_{x, y ; s, t}(\tau)=A_{1}\left(\gamma_{x, y ; s, t}(\tau)\right)\left(\dot{\gamma}_{x, y ; s, t}\right)_{1}(\tau)+A_{2}\left(\gamma_{x, y ; s, t}(\tau)\right)\left(\dot{\gamma}_{x, y ; s, t}\right)_{2}(\tau), \quad 0 \leqslant \tau \leqslant 1,
$$

and, for $k \geqslant 1$, define 


$$
J_{k}(x, y ; s, t)=\int_{0}^{1} \int_{0}^{\tau_{1}} \cdots \int_{0}^{\tau_{k-1}} B_{x, y ; s, t}\left(\tau_{1}\right) B_{x, y ; s, t}\left(\tau_{2}\right) \cdots B_{x, y: s . t}\left(\tau_{k}\right) d \tau_{k} \cdots d \tau_{2} d \tau_{1}
$$

Then, applying Theorem 4.3 in Sec. 1.4 of Ref. 7 (p. 31), we have for all $s, t \in \mathbf{R}$,

$$
W_{s, t}^{A}(x, y)=I+\sum_{k=1}^{\infty}(-1)^{k} J_{k}(x, y ; s, t), \quad(x, y) \in M_{s, t} .
$$

There is a positive constant $t_{0}<1$, such that for all $|t| \in\left(0, t_{0}\right), \mathbf{a}_{n} \notin D_{x, y ; t, t}, n=1, \ldots, N$. Let $0<|t|<t_{0}$ and $a=\sup \left\{\left\|A_{j}\left(\mathbf{r}^{\prime}\right)\right\| ; \mathbf{r}^{\prime} \in D_{x, y ; t_{0}, t_{0}} \cup D_{x, y ;-t_{0},-t_{0}}, j=1,2\right\}$. Then we have $\left\|B_{x, y ; t, t}(\tau)\right\| \leqslant 8 a|t|$, which implies that $\left\|J_{k}(x, y ; t, t)\right\| \leqslant(8 a|t|)^{k} / k !, k \geqslant 1$. Hence, we have

$$
\sum_{k=3}^{\infty}\left\|J_{k}(x, y ; t, t)\right\| \leqslant C t^{3}
$$

where $C=\sum_{k=3}^{\infty}(8 a)^{k} / k !<\infty$. By Stokes' theorem, we have

$$
J_{1}(x, y ; t, t)=\int_{D_{x, y ; f, t}}\left(\partial_{1} A_{2}\left(\mathbf{r}^{\prime}\right)-\partial_{2} A_{1}\left(\mathbf{r}^{\prime}\right)\right) d \mathbf{r}^{\prime}
$$

We can write

$$
J_{2}(x, y ; t, t)=\frac{1}{2}\left(J_{+}+J_{-}\right)
$$

where

$$
J_{ \pm}=\int_{0}^{1} \int_{0}^{\tau_{1}}\left(B_{x, y ; t, t}\left(\tau_{1}\right) B_{x, y ; t, t}\left(\tau_{2}\right) \pm B_{x, y ; t, t}\left(\tau_{2}\right) B_{x, y ; t, t}\left(\tau_{1}\right)\right) d \tau_{2} d \tau_{1}
$$

By symmetry, we have $J_{+}=J_{1}(x, y ; t, t)^{2} / 2$. By (3.6), we have $\left\|J_{1}(x, y ; t, t)\right\| \leqslant b t^{2}, 0<|t|<t_{0}$, where $b=\sup \left\{\left\|\partial_{1} A_{2}\left(\mathbf{r}^{\prime}\right)-\partial_{2} A_{1}\left(\mathbf{r}^{\prime}\right)\right\| ; \mathbf{r}^{\prime} \in D_{x, y ; t_{0}, t_{0}} \cup D_{x, y ;-t_{0},-t_{0}}\right\}$. Hence,

$$
\left\|J_{+}\right\| \leqslant \frac{b^{2}}{2} t^{4}
$$

To estimate $J_{-}$, we note that

$$
B_{x, y: t, t}(\tau)=A_{1}(x, y)\left(\dot{\gamma}_{x, y ; t, t}\right)_{1}(\tau)+A_{2}(x, y)\left(\dot{\gamma}_{x, y ; t, t}\right)_{2}(\tau)+O\left(t^{2}\right)
$$

as $t \rightarrow 0$ uniformly in $\tau \in[0,1]$. Hence,

$$
\begin{aligned}
& B_{x, y ; t, t}\left(\tau_{1}\right) B_{x, y ; t, t}\left(\tau_{2}\right)-B_{x, y ; t, t}\left(\tau_{2}\right) B_{x, y ; t, t}\left(\tau_{1}\right) \\
& \quad=\sum_{\mu, \nu=1}^{2}\left[A_{\mu}(x, y), A_{\nu}(x, y)\right]\left(\dot{\gamma}_{x, y ; t, t}\right)_{\mu}\left(\tau_{1}\right)\left(\dot{\gamma}_{x, y ; t, t}\right)_{\nu}\left(\tau_{2}\right)+O\left(|t|^{3}\right) .
\end{aligned}
$$

It follows that 


$$
\begin{aligned}
J_{-} & =-\left[A_{1}(x, y), A_{2}(x, y)\right] \int_{C_{x, y ; t, t}}\left(x^{\prime} d y^{\prime}-y^{\prime} d x^{\prime}\right)+O\left(|t|^{3}\right) \\
& =-2 \int_{D_{x, y ; t, t}}\left[A_{1}\left(\mathbf{r}^{\prime}\right), A_{2}\left(\mathbf{r}^{\prime}\right)\right] d \mathbf{r}^{\prime}+O\left(|t|^{3}\right) .
\end{aligned}
$$

Substituting (3.5)-(3.8) into (3.4), we obtain (3.3).

The following lemma is well known. ${ }^{11}$

Lemma 3.3: Suppose that $A$ is flat on $M$ and each component $A_{j}$ is $m$ times continuously differentiable on $M$. Then, for every simply connected domain $D$ of $M$, there exists a $U(p)$ valued, $m+1$ times continuously differentiable function $g$ on $D$, such that $A_{j}=g^{-1} \partial_{j} g$ on $D$.

Lemma 3.4: Suppose that $A$ is flat on $M$. Then the following (i)-(iii) hold.

(i) Let $C$ be any continuous, piecewise continuously differentiable closed path in $M$, which is contractible to a point within $M$. Then

$$
W_{A}(C)=I \text {. }
$$

(ii) Let $C_{\epsilon}^{\mathbf{r}_{1}}\left(\mathbf{a}_{n}\right) \subset D_{x, y ; s, t}$, with $\left|\mathbf{r}_{1}-\mathbf{a}_{n}\right|=\epsilon<\delta_{0}$ and $D_{x, y ; s, t} \cap\left\{\mathbf{a}_{1}, \ldots, \mathbf{a}_{N}\right\}=\left\{\mathbf{a}_{n}\right\}$. Then there exists a unitary matrix $U$, such that

$$
W_{s, t}^{A}(x, y)=U W_{A}\left(C_{\epsilon}^{\mathbf{r}_{1}}\left(\mathbf{a}_{n}\right)\right) U^{-1} .
$$

(iii) Let $0<\epsilon_{1}<\epsilon_{2}<\delta_{0}$ and $\mathbf{r}_{j} \in \mathbf{R}^{2}, j=1,2$, be, such that $\left|\mathbf{r}_{j}-\mathbf{a}_{n}\right|=\epsilon_{j}$. Then

$$
W_{A}\left(C_{\epsilon_{2}}^{\mathbf{r}_{2}}\left(\mathbf{a}_{n}\right)\right)=W_{A}\left(L_{\mathbf{r}_{2} ; \mathbf{r}_{1}}\right)^{-1} W_{A}\left(C_{\epsilon_{1}}^{\mathbf{r}_{1}}\left(\mathbf{a}_{n}\right)\right) W_{A}\left(L_{\mathbf{r}_{2} ; \mathbf{r}_{1}}\right),
$$

where $L_{\mathbf{r}_{2} ; \mathbf{r}_{1}}$ denotes the straight line from $\mathbf{r}_{2}$ to $\mathbf{r}_{1}$.

Proof: (i) The path $C$ is included in a simply connected domain $D$ of $M$. By Lemma 3.3, there exists a $U(p)$-valued twice differentiable function $g$ on $D$, such that $A_{j}=g^{-1} \partial_{j} g$ on $D(j=1,2)$. In terms of $h:=g^{-1}$, we can write $A_{j}=-\left(\partial_{j} h\right) h^{-1}$. Let $\gamma:[0,1] \rightarrow M$ be a parametrization of $C$ $(x(0)=x(1))$. Then we have

$$
-\left\{A_{1}(\gamma(\tau)) \dot{\gamma}_{1}(\tau)+A_{2}(\gamma(\tau)) \dot{\gamma}_{2}(\tau)\right\}=\frac{d h(\gamma(\tau))}{d \tau} h(\gamma(\tau))^{-1}
$$

Hence, applying Theorem 3.1 on p. 20 in Ref. 7, we have $W_{A}(C)=h(\gamma(1)) h(\gamma(0))^{-1}=I$. Thus we obtain (3.9).

(ii) We decompose $D_{x, y ; s, t}$, as is shown in Fig. 1.

Let $V_{j}=W_{A}\left(C_{j}\right), W_{j}=W_{A}\left(L_{j}\right), j=1,2$. Then, by part (i), we have

$$
W_{2} V_{1} W_{1} W_{s, i}^{A,-}(x, y)=I, \quad W_{1}^{-1} V_{2}^{-1} W_{2}^{-1}\left(W_{s, t}^{A,+}\right)^{-1}=I,
$$

which imply $W_{2} V_{1} V_{2}^{-1} W_{2}^{-1} W_{s, t}^{A}(x, y)=I$. We have $V_{2} V_{1}^{-1}=W_{A}\left(C_{\epsilon}^{\mathbf{r}_{1}}\left(\mathbf{a}_{n}\right)\right)$. Thus, taking $U=W_{2}$, we obtain (3.10).

(iii) We need only repeat the proof of part (ii) with $\epsilon$ and $D_{x, y ; s, t}$ replaced by $\epsilon_{1}$ and $C_{\epsilon_{2}}^{\mathbf{r}_{2}}\left(\mathbf{a}_{n}\right)$, respectively.

Proof of Theorem 3.1: Suppose that $W_{s, t}^{A}-I$ holds for all $s, t \in \mathbf{R}$. Then Lemma 3.2 implies that $F_{12}(\mathbf{r})=0$ for all $\mathbf{r} \in M$. Hence, $A$ is flat on $M$. Then, using Lemma 3.4 (i) and (ii), we obtain (3.2).

Conversely, suppose that $A$ is flat on $M$ and (3.2) holds. Then, by Lemma 3.4(i), we have $W_{s, t}^{A}(x, y)=I$ for all $C_{x, y ; s, t}$ contractible to a point within $M$. Let $\mathbf{a}_{n} \in D_{x, y ; s, t}$, but $\mathbf{a}_{m} \notin D_{x, y ; s, t}$ for $m \neq n$. Then, by Lemma 3.4 (ii), we have 


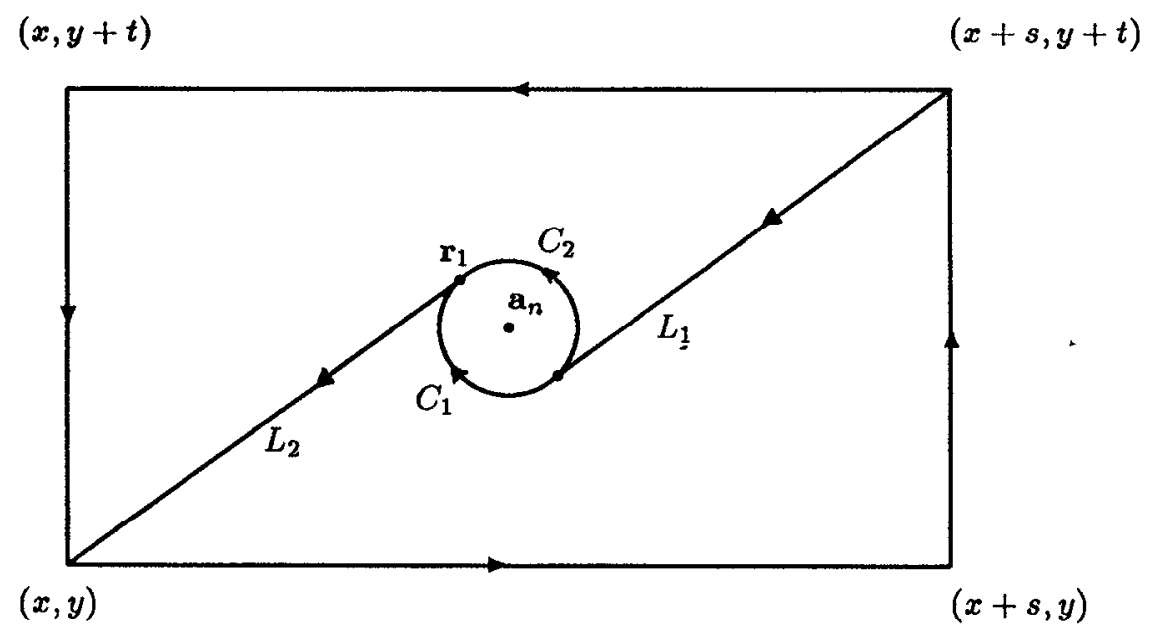

FIG. 1. The decomposition of $D_{x, y ; s, t}$ for proof of Lemma 3.4 (ii).

$$
W_{s, t}^{A}(x, y)=U W_{A}\left(C_{\epsilon}^{\mathbf{r}_{1}}\left(\mathbf{a}_{n}\right)\right) U^{-1}=U I U^{-1}=I .
$$

Hence, in this case, $W_{s, t}^{A}(x, y)=I$. Finally, we consider the case where $D_{x, y ; s, t}$ includes $\left\{\mathbf{a}_{i_{1}}, \ldots, \mathbf{a}_{i_{k}}\right\}$, where $2 \leqslant k \leqslant N$ and $\left\{i_{1}, \ldots, i_{k}\right\}$ is a subset of $\{1, \ldots, N\}$. In this case we decompose $D_{x, y ; s, t}$, as is shown in Fig. 2, where we set $\mathbf{b}_{j}=\mathbf{a}_{i_{j}}, j=1, \ldots, k$.

Then, by Lemma 3.4(ii), there exist $U_{j} \in U(p), j=1, \ldots, k$, such that for $j=1, \ldots, k$,

$$
U_{j} W_{A}\left(C_{\epsilon}^{\mathbf{r}_{j}}\left(\mathbf{b}_{j}\right)\right) U_{j}^{-1}=W_{A}\left(D_{j-1}^{-1} \circ F_{j}^{\circ} \circ D_{j}^{\circ} C_{j}\right) .
$$

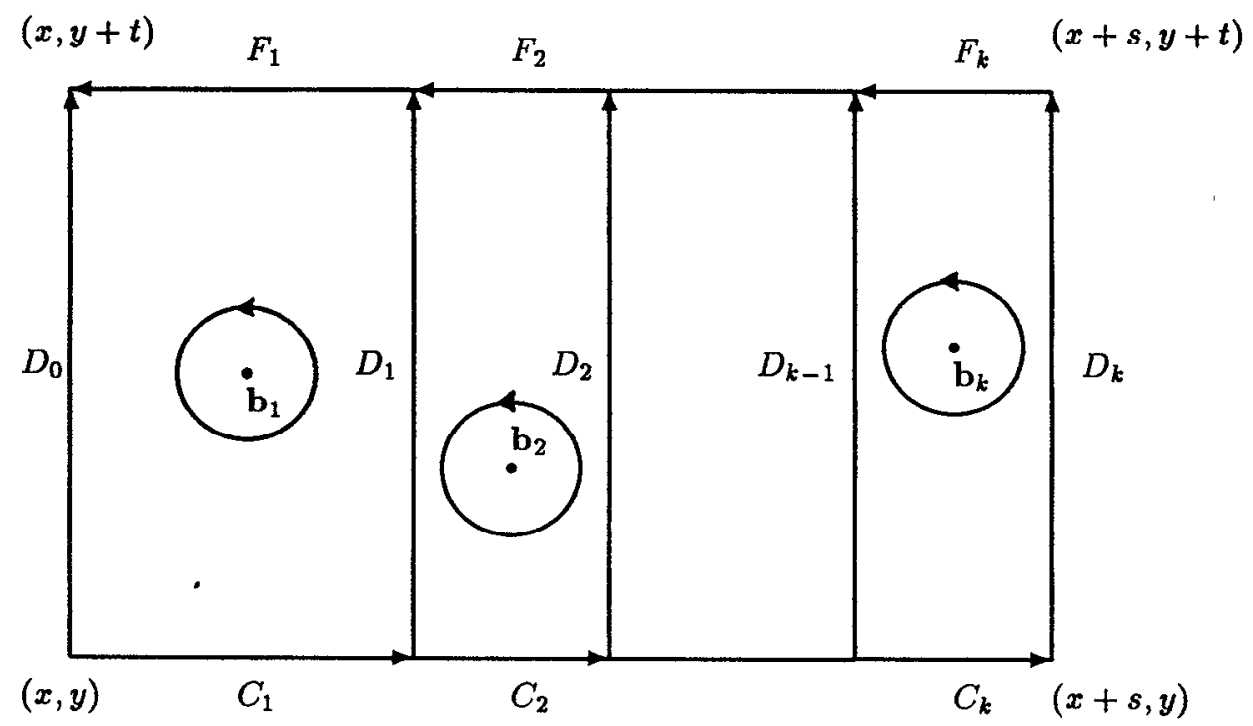

FIG. 2. The decomposition of $D_{x, y ; s, t}$ for proof of Theorem 3.1. 
By (3.2), each of the RHSs of these equalities turns out to be the identity. Then the resulting equalities give $W_{s, t}^{A}(x, y)=I$.

\section{ESSENTIAL SELF-ADJOINTNESS OF $\boldsymbol{P}_{j}$ AND NON-SCHRÖDINGER REPRESENTATIONS}

For $j=1,2$, we set $S_{j}=\mathbf{R} \backslash\left\{a_{n j}\right\}_{n=1}^{N}$. Let

$$
\mathscr{Z}_{1}^{m}=C_{0}^{m}\left(\mathbf{R} \times S_{2} ; \mathbf{C}^{p}\right), \quad \mathscr{Z}_{2}^{m}=C_{0}^{m}\left(S_{1} \times \mathbf{R} ; \mathbf{C}^{p}\right)
$$

For a subset $V$ of $M_{p}(\mathbf{C})$ (the set of $p \times p$ complex matrices) and an open subset $D$ of $M$, we denote by $C^{m}(D ; V)$ the set of $V$-valued, $m$ times continuously differentiable functions on $D$. We introduce a class of gauge potentials.

Definition 4.1: We say that a 1 -form $A$ on $M$ is in the set $\mathfrak{A}_{m}$ if there exist $g_{1} \in C^{m+1}\left(\mathbf{R} \times S_{2} ; U(p)\right)$ and $g_{2} \in C^{m+1}\left(S_{1} \times \mathbf{R} ; U(p)\right)$, such that

$$
A_{1}=g_{1}^{-1} \partial_{1} g_{1}, \quad \text { on } \mathbf{R} \times S_{2} ; \quad A_{2}=g_{2}^{-1} \partial_{2} g_{2}, \quad \text { on } S_{1} \times \mathbf{R} \text {. }
$$

Theorcm 4.2: Assume that $\Lambda \in \mathfrak{A}_{m-1}(m \geqslant 1)$. Then, each $P_{j}$ is essentially self-adjoint on $\mathscr{Z}_{j}^{m}$.

Proof: Let $g_{j}$ be as in Definition 4.1. Then $g_{j}$ is a bijective mapping from $\mathscr{L}_{j}^{m}$ to itself; we have $P_{j} \psi=g_{j}^{-1} p_{j} g_{j} \psi, \psi \in \mathscr{Z}_{j}^{m}$. Hence, we need only to show that $p_{j}$ is essentially self-adjoint on $\mathcal{Z}_{j}^{m}$. But this is a well-known fact (see the proof of Theorem 3.2 in Ref. 1).

Theorem 4.3: Suppose that $A_{j} \in C^{m}\left(M ; M_{p}^{\mathrm{ah}}(\mathbf{C})\right)(j=1,2)(m \geqslant 1)$ and $A=A_{1} d x+A_{2} d y$ is flat on $M$. Then $A \in \mathfrak{A}_{m}$. In particular, $P_{j}$ is essentially self-adjoint on $Z_{j}^{m+1}$.

Proof: Let $b_{1}, \ldots, b_{k}(k \leqslant N)$ be the numbers different from each other in the set $\left\{a_{n 2}\right\}_{n=1}^{N}$, with $b_{1}<b_{2}<\cdots<b_{k}$. Let $M_{l}=\left\{(x, y) \in \mathbf{R}^{2} \mid b_{l-1}<y<b_{l}\right\}, l=1, \ldots, k+1$, with $b_{0}=-\infty$, $b_{k+1}=+\infty$. Then, each $M_{l}$ is simply connected and $\mathbf{R} \times S_{2}=\cup_{l=1}^{k+1} M_{l}$. By Lemma 3.3, there exists a function $h_{l} \in C^{m+1}\left(M_{l} ; U(p)\right)$, such that $A_{j}=h_{l}^{-1} \partial_{j} h_{l}(j=1,2)$ on $M_{l}$. Defining $g_{1} \in C^{m+1}\left(\mathbf{R} \times S_{2} ; U(p)\right)$ by $g_{1}(\mathbf{r})=h_{l}(\mathbf{r})$ if $\mathbf{r} \in M_{l}$, we have $A_{j}=g_{1}^{-1} \partial_{j} g_{1}$ on $\mathbf{R} \times S_{2}$. In particular, $A_{1}=g_{1}^{-1} \partial_{1} g_{1}$ on $\mathbf{R} \times S_{2}$. Similarly, we can show that there exists a function $g_{2} \in C^{m+1}\left(S_{1} \times \mathbf{R} ; U(p)\right)$, such that $A_{2}=g_{2}^{-1} \partial_{2} g_{2}$ on $S_{1} \times \mathbf{R}$.

Corollary 2.5 and Theorems 3.1 and 4.3 yield the following result.

Theorem 4.4: Suppose that $A_{j} \in C^{m}\left(M ; M_{p}^{\text {ah }}(\mathbf{C})\right)(j=1,2)(m \geqslant 1)$ and $A$ is flat on $M$. Then, the representation $\left\{\bar{P}_{j}, q_{j}\right\}_{j=1}^{2}$ of CCR is a Schrödinger 2-system if and only if there exists a constant $\delta \in\left(0, \delta_{0}\right)$, such that for all $\epsilon<\delta$ and some $\mathbf{r}_{n} \in M$ with $\left|\mathbf{r}_{n}-\mathbf{a}_{n}\right|=\epsilon, W_{A}\left(C_{\epsilon}^{\mathbf{r}_{n}}\left(\mathbf{a}_{n}\right)\right)=I$, $n=1, \ldots, N$.

This theorem also gives a characterization for $\left\{\vec{P}_{j}, q_{j}\right\}_{j=1}^{2}$ to be non-Schrödinger.

\section{EXAMPLE}

In this section we discuss a class of $M_{p}^{\text {ah }}(\mathbf{C})$-valued, flat 1-forms on $M$. Let $S_{n}$ and $T_{n}$ be $p \times p$ Hermitian constant matrices, such that, for all $n \neq m(n, m=1, \ldots, N)$,

$$
\left[S_{n}, S_{m}\right]=0, \quad\left[T_{n}, T_{m}\right]=0, \quad\left[S_{n}, T_{m}\right]=0,
$$

but $S_{n}$ does not necessarily commute with $T_{n}$. Let $\phi_{n}$ be a real-valued, continuously differentiable function on $\mathbf{R}^{2}(n=1, \ldots, N)$ (so that $\phi_{n}$ and $\partial_{j} \phi_{n}$ have no singularity at $\left.\mathbf{r}=\mathbf{a}_{n}, n=1, \ldots, N\right)$. Then, for each $\mathbf{r} \in M, e^{ \pm i S_{n} \phi_{n}(\mathbf{r})}$ are in $U(p)$ and the matrix

$$
K_{n}(\mathbf{r}):=e^{-i S_{n} \phi_{n}(\mathbf{r})} T_{n} e^{i S_{n} \phi_{n}(\mathbf{r})}
$$

is Hermitian. Hence 


$$
A_{1}(\mathbf{r}):=-i \sum_{n=1}^{N}\left\{\frac{\left(y-a_{n 2}\right)}{\left|\mathbf{r}-\mathbf{a}_{n}\right|^{2}} K_{n}(\mathbf{r})-S_{n} \partial_{1} \phi_{n}(\mathbf{r})\right\}
$$

and

$$
A_{2}(\mathbf{r}):=i \sum_{n=1}^{N}\left\{\frac{\left(x-a_{n 1}\right)}{\left|\mathbf{r}-\mathbf{a}_{n}\right|^{2}} K_{n}(\mathbf{r})+S_{n} \partial_{2} \phi_{n}(\mathbf{r})\right\}
$$

are in $M_{p}^{\text {ah }}(\mathrm{C})$. It is easy to see that $A_{1}$ and $A_{2}$ satisfy the nonlinear partial differential equation,

$$
\partial_{1} A_{2}(\mathbf{r})-\partial_{2} A_{1}(\mathbf{r})+\left[A_{1}(\mathbf{r}), A_{2}(\mathbf{r})\right]=2 \pi i \sum_{n=1}^{N} K_{n}\left(\mathbf{a}_{n}\right) \delta\left(\mathbf{r}-\mathbf{a}_{n}\right)
$$

where $\delta(\mathbf{r})$ is the two-dimensional Dirac delta distribution. Hence, the 1-form $A=A_{1} d x+A_{2} d y$ is flat on $M$. For $p \geqslant 2$, this example is a non-Abelian generalization of examples in Ref. 1 and Ref. 3.

We want to compute the Wilson loop $W_{A}\left(C_{\epsilon}^{\mathrm{r}}\left(\mathbf{a}_{n}\right)\right)$ in the present case. We first note that we can write $A_{j}$ in the form

$$
A_{1}(\mathbf{r})=-\frac{i\left(y-a_{n 2}\right)}{\left|\mathbf{r}-\mathbf{a}_{n}\right|^{2}} K_{n}\left(\mathbf{a}_{n}\right)+F_{n}^{(1)}(\mathbf{r}), \quad A_{2}(\mathbf{r})=\frac{i\left(x-a_{n 1}\right)}{\left|\mathbf{r}-\mathbf{a}_{n}\right|^{2}} K_{n}\left(\mathbf{a}_{n}\right)+F_{n}^{(2)}(\mathbf{r}),
$$

where $F_{n}^{(j)}(j=1,2)$ is a function continuous in the domain

$$
D_{n}=\left\{\mathbf{r} \in \mathbf{R}^{2}|0<| \mathbf{r}-\mathbf{a}_{n} \mid<\delta\right\}
$$

$\left(\delta<\delta_{0}\right)$ with $\sup _{\mathbf{r} \in D_{n}}\left\|F_{n}^{(j)}(\mathbf{r})\right\|<\infty, j=1,2$.

Lemma 5.1: Let $B=B_{1} d x+B_{2} d y$ be an $M_{p}(\mathrm{C})$-valued, continuously differentiable 1-form on $M$. Suppose that there exist a constant matrix $S \in M_{p}(\mathbf{C})$ and $M_{p}(\mathbf{C})$-valued continuous functions $G_{1}, G_{2}$ on $D_{n}$, such that $C_{j}:=\sup _{\mathbf{r} \in D_{n}}\left\|G_{j}(\mathbf{r})\right\|<\infty, j=1,2$, and, for all $\mathbf{r} \in D_{n}$,

$$
B_{1}(\mathbf{r})=-\frac{i\left(y-a_{n 2}\right)}{\left|\mathbf{r}-\mathbf{a}_{n}\right|^{2}} S+G_{1}(\mathbf{r}), \quad B_{2}(\mathbf{r})=\frac{i\left(x-a_{n 1}\right)}{\left|\mathbf{r}-\mathbf{a}_{n}\right|^{2}} S+G_{2}(\mathbf{r})
$$

Then

$$
\lim _{\epsilon \downarrow 0} W_{B}\left(C_{\epsilon}^{\mathbf{r}}\left(\mathbf{a}_{n}\right)\right)=e^{-2 \pi i S},
$$

independently of the choice of the initial point $\mathbf{r}$, with $\left|\mathbf{r}-\mathbf{a}_{n}\right|=\epsilon$.

Proof: We parametrize the circle $C_{\epsilon}^{\mathbf{r}}\left(\mathbf{a}_{n}\right)$ by $\chi(\theta)=\mathbf{a}_{n}+\left(\epsilon \cos \left(\theta+\theta_{0}\right), \epsilon \sin \left(\theta+\theta_{0}\right)\right), 0 \leqslant \theta \leqslant 2 \pi$, where $\mathbf{r}=\mathbf{a}_{n}+\left(\epsilon \cos \theta_{0}, \epsilon \sin \theta_{0}\right)(\epsilon<\delta)$. Then we have

$$
B_{1}(\gamma(\theta)) \dot{\gamma}_{1}(\theta)+B_{2}(\gamma(\theta)) \dot{\gamma}_{2}(\theta)=i S+\epsilon F(\epsilon, \theta),
$$

where $F(\epsilon, \theta)=G_{2}(\mathcal{\gamma}(\theta)) \cos \left(\theta+\theta_{0}\right)-G_{1}(\chi(\theta)) \sin \left(\theta+\theta_{0}\right)$. Hence, we have

$$
W_{B}\left(C_{\epsilon}^{\mathrm{r}}\left(\mathbf{a}_{n}\right)\right)=\lim _{m \rightarrow \infty} e^{-2 \pi\left[i S+\epsilon F\left(\epsilon, \theta_{m}\right)\right] / m} e^{-2 \pi\left[i S+\epsilon F\left(\epsilon, \theta_{m-1}\right)\right] / m} \cdots e^{-2 \pi\left[i S+\epsilon F\left(\epsilon, \theta_{1}\right)\right] / m},
$$


where $\theta_{j}=2 \pi j / m, j=1, \ldots, m$. By the condition for $G_{j}, j=1,2$, we have $\|F(\epsilon, \theta)\| \leqslant C$, where $C=C_{1}+C_{2}$. In general, we can show that, for all $M_{j}, N_{j} \in M_{p}(\mathbf{C}), j=1, \ldots, k(k=1,2, \ldots)$,

$$
\left\|e^{M_{1}+N_{1}} \cdots e^{M_{k}+N_{k}}-e^{M_{1}} \cdots e^{M_{k}}\right\| \leqslant\left(\sum_{j=1}^{k}\left\|N_{j}\right\|\right) \exp \left(2 \sum_{j=1}^{k}\left\|M_{j}\right\| e \sum_{j=1}^{k}\left\|N_{j}\right\|\right) .
$$

Applying this estimate to $M_{j}=2 \pi i S / m, N_{j}=2 \pi \epsilon F\left(\epsilon, \theta_{j}\right) / m$, we obtain

$$
\left\|e^{-2 \pi\left[i S+\epsilon F\left(\epsilon, \theta_{m}\right)\right] / m} e^{-2 \pi\left[i S+\epsilon F\left(\epsilon, \theta_{m-1}\right)\right] / m} \cdots e^{-2 \pi\left[i S+\epsilon F\left(\epsilon, \theta_{1}\right)\right] / m}-e^{-2 \pi i S}\right\| \leqslant C^{\prime} \epsilon,
$$

with $C^{\prime}=2 \pi C \exp (4 \pi\|S\|+2 \pi C \epsilon)$. Hence, $\left\|W_{B}\left(C_{\epsilon}^{\mathrm{r}}\left(\mathbf{a}_{n}\right)\right)-e^{-2 \pi i S}\right\| \leqslant C^{\prime} \epsilon$, which implies the desired result.

Applying Lemma 5.1 to the present example, we obtain the following.

Lemma 5.2: For all $n=1, \ldots, N$,

$$
\lim _{\epsilon \downarrow 0} W_{A}\left(C_{\epsilon}^{\mathbf{r}}\left(\mathbf{a}_{n}\right)\right)=e^{-2 \pi i K_{n}\left(\mathbf{a}_{n}\right)},
$$

independently of the choice of the initial point $\mathbf{r}$ with $\left|\mathbf{r}-\mathbf{a}_{n}\right|=\epsilon$.

Lemma 5.3: Let $0<\epsilon_{1}<\epsilon_{2}<\delta_{0}$ and $\mathbf{r}_{j} \in \mathbf{R}^{2}, j=1,2$, be such that $\left|\mathbf{r}_{j}-\mathbf{a}_{n}\right|=\epsilon_{j}$ and $\mathbf{r}_{1}-\mathbf{a}_{n}=\alpha\left(\mathbf{r}_{2}-\mathbf{r}_{1}\right)$ with a constant $\alpha>0$. Then

$$
W_{A}\left(L_{\mathbf{r}_{2} ; \mathbf{a}_{n}}\right):=\lim _{\epsilon_{1} \downarrow 0} W_{A}\left(L_{\mathbf{r}_{2} ; \mathbf{r}_{1}}\right)
$$

exists.

Proof: The straight line $L_{\mathbf{r}_{2} ; \mathbf{a}_{n}}$ is parametrized by $l(\tau)=(1-\tau) \mathbf{r}_{2}+\tau \mathbf{a}_{n}, 0 \leqslant \tau \leqslant 1$. There exists a number $\tau_{1} \in(0,1)$, such that $\mathbf{r}_{1}=l\left(\tau_{1}\right)$. Then we have, for $\tau \in[0,1)$,

$$
A_{1}(l(\tau)) \dot{l}_{1}(\tau)+A_{2}(l(\tau)) \dot{l}_{2}(\tau)=f_{n}(\tau)
$$

where $f_{n}(\tau)=\left(F_{n}^{(1)}(l(\tau)), F_{n}^{(2)}(l(\tau))\right) \cdot\left(\mathbf{a}_{n}-\mathbf{r}_{2}\right)$. It is easy to see that $C_{n}:=\lim _{\tau \uparrow 1} f_{n}(\tau)$ exists. Hence $f_{n}$ can be extended to a continuous function on $[0,1]$ with $f_{n}(1)=C_{n}$. We have $W_{A}\left(L_{\mathbf{r}_{2} ; \mathbf{r}_{1}}\right)=\Pi_{0}^{\tau_{1}} e^{-f_{n}(\tau) d \tau}$. On the other hand, $\Pi_{0}^{t} e^{-f_{n}(\tau) d \tau}$ is continuous in $t \in[0,1]$. Thus, the desired result follows.

Lemma 5.4: Let $0<\delta<\delta_{0}$ be fixed. Then, for all $\epsilon \in(0, \delta), n=1, \ldots, N$, and all $\mathbf{r} \in M$ with $\left|\mathbf{r}-\mathbf{a}_{n}\right|=\epsilon$,

$$
W_{A}\left(C_{\epsilon}^{\mathbf{r}}\left(\mathbf{a}_{n}\right)\right)=W_{A}\left(L_{\mathbf{r} ; \mathbf{a}_{n}}\right)^{-1} e^{-2 \pi i K_{n}\left(\mathbf{a}_{n}\right)} W_{A}\left(L_{\mathbf{r} ; \mathbf{a}_{n}}\right)
$$

Proof: By Lemmas 5.2 and 5.3, we can take the limit $\epsilon_{1} \downarrow 0$ of the RHS of (3.11) to obtain

$$
W_{A}\left(C_{\epsilon_{2}}^{\mathbf{r}_{2}}\left(\mathbf{a}_{n}\right)\right)=W_{A}\left(L_{\mathbf{r}_{2} ; \mathbf{a}_{n}}\right)^{-1} e^{-2 \pi i K_{n}\left(\mathbf{a}_{n}\right)} W_{A}\left(L_{\mathbf{r}_{2} ; \mathbf{a}_{n}}\right)
$$

Thus, the desired result follows.

By Lemma 5.4 and Theorem 4.4, we obtain the following theorem.

Theorem 5.5: In the present example, the representation $\left\{\bar{P}_{j}, q_{j}\right\}_{j=1}^{2}$ of CCR is a Schrödinger 2-system if and only if, for all $n=1, \ldots, N$, all the eigenvalues of $T_{n}$ are integers. 
Proof: We need only consider the condition that $e^{-2 \pi i K_{n}\left(\mathbf{a}_{n}\right)}=I$ for all $n=1, \ldots, N$, which is equivalent to the condition that $e^{2 \pi i T_{n}}=I$ for all $n=1, \ldots, N$ [note that $K_{n}\left(\mathbf{a}_{n}\right)$ is unitarily equivalent to $\left.T_{n}\right]$. Since $T_{n}$ is Hermitian, $e^{2 \pi i T_{n}}=I$ if and only if all the eigenvalues of $T_{n}$ are integers.

Theorem 5.5 implies the following: Let

$$
\mathfrak{A}=\left\{A=A_{1} d x+A_{2} d y \mid \text { at least one } T_{n} \text { has a non-integer eigenvalue }\right\} .
$$

Then, for each $A \in \mathfrak{A},\left\{\bar{P}_{j}, q_{j}\right\}_{j=1}^{2}$ is a non-Schrödinger representation of the CCR, with two degrees of freedom. Thus, we obtain a class of non-Schrödinger representations of CCR associated with $M_{p}^{\text {ah }}(\mathbf{C})$-valued, flat 1 -forms on $M$.

\section{ACKNOWLEDGMENT}

This work is supported by the Grant-In-Aid No. 06640188 for science research from the Ministry of Education, Japan.

${ }^{\text {t}}$ A. Arai, "Momentum operators with gauge potentials, local quantization of magnetic flux, and representation of canonical commutation relations," J. Math. Phys. 33, 3374-3378 (1992).

${ }^{2}$ C. R. Putnam, Commutation Properties of Hilbert Space Operators (Springer, Berlin, 1967).

${ }^{3}$ H. Reeh, "A remark concerning canonical commutation relations," J. Math. Phys. 29, 1535-1536 (1988).

${ }^{4}$ Y. Aharonov and D. Bohm, "Significance of electromagnetic potentials in the quantum theory," Phys. Rev. 115, 485-491 (1959).

${ }^{5}$ A. Arai, "Properties of the Dirac-Weyl operator with a strongly singular gauge potential," J. Math. Phys. 34, 915-935 (1993).

${ }^{6} \mathrm{H}$. Kurose and H. Nakazato, "Geometric construction of *-representations of the Weyl algebra with degree 2," preprint, 1994.

${ }^{7}$ J. D. Dollard and C. N. Friedman, "Product integration," Encyclopedia of Mathematics and Its Applications Vol. 10: Analysis (Addison-Wesley, Reading, Massachusetts, 1979).

${ }^{8}$ In the notation used in physics, $W_{A}(C)=P \exp \left(-\int_{C} A\right)$.

${ }^{9} \mathrm{~J}$. von Neumann, "Die Eindeutigkeit der Schrödingerschen Operatoren," Math. Ann. 104, $570-578$ (1931).

${ }^{10} \mathrm{M}$. Göckeler and T. Schücker, Differential Geometry, Gauge Theories, and Gravity (Cambridge U.P., Cambridge, 1987).

${ }^{11}$ L. Schlesinger, "Wcitcre Bciträge zum Infinitesimalkalkul der Matrizen," Math. Zeit. 35, 485-501 (1932). 